\title{
DESIGN OF A NEW SCALE TO MEASURE THE LEARNER EXPERIENCE IN E-LEARNING SYSTEMS
}

\author{
Yassine Safsouf ${ }^{1,3}$, Khalifa Mansouri ${ }^{2}$ and Franck Poirier ${ }^{1}$ \\ ${ }^{1}$ Lab-STICC, University Bretagne Sud, France \\ ${ }^{2}$ Laboratory SSDIA, ENSET of Mohammedia, University Hassan II of Casablanca, Morocco \\ ${ }^{3}$ LIMIE Laboratory, ISGA Group, Centre Marrakech, Morocco
}

\begin{abstract}
Nowadays, the user experience (UX) has become an essential concept of human-computer interaction (HCI) and occupies an increasingly important place in companies interested in this field. Designing a system or product with a good user experience is one of the main objectives of any digital project. E-learning systems must adapt because, beyond the benefits associated with good usability, creating a learning system with a better user experience (learner's experience) is a key competitive factor in the marketplace. This paper describes a new measurement tool based on a theoretical model presented in previous research that distinguishes the success factors of learners in an e-learning system. This tool for evaluating the learner experience is based on the AttrakDiff user experience measurement tool and uses the same subscale, namely the pragmatic and hedonic qualities of interactive systems. The proposed learning experience (LX) scale allow to know if a e-learning system is perceived by learners as interactive, effective, clear, reliable, challenging, or effortless.
\end{abstract}

\section{KEYWORDS}

Learner Experience, Learner Satisfaction, Learner Success, Usability, User Experience, AttrakDiff

\section{INTRODUCTION}

Today, information and communication technologies (ICTs) are of fundamental importance in society and must therefore be considered as a strategic sector that contributes significantly to economic and social development. These technologies are evolving at an accelerated rate. Their rapid obsolescence requires a constant updating of knowledge and know-how. Accordingly, the teaching and learning in universities are adapted to keep up with the changes in ICTs for the development of quality education.

The web-based technologies have been used as a powerful tool to increase the accessibility to education for people around the world. They provide now to the learners, the opportunity to access the information they need at the right time, and also online educational approaches to conduct distance teaching and learning. Nevertheless, it is not obvious how to measure the improvement of the learning process, for example are they related to learner characteristics, instructor, technology, course contents or social interactions?

Although the learner's experience (LX) has been the subject of several previous studies (Sudhakar A., 2015), it has been limited to certain concerns, such as student perceptions or friendliness. We have discussed and classified in previous articles models and factors for evaluating LX in a distance learning system (Safsouf, Mansouri and Poirier, 2017) (Safsouf, Mansouri and Poirier, 2018), such as learner characteristics, instructor characteristics, system characteristics, course characteristics and social aspects.

In this regard, this paper is an attempt to propose a new scale for measuring the learning experience in an e-learning system. The rest of this paper is organised as follows: section II discusses the concepts of usability and user experience with its roots and defines the term "learner experience"; section III provides a tool to evaluate learners' experience in an e-learning system and section IV concludes the paper. 


\section{RELATED WORK}

The usability of a system refers in a broad meaning to its ease of learning and efficiency. This concept has become much more popular with the emergence of computer or interactive systems for a wider audience, but also because of increased competition between companies. Improving the usability of a system reduces user frustration and makes it easier and faster to complete the task. Shackel and Richardson (Shackel, B., Richardson, 1991) was among the first authors to focus on usability, for them the precise definition of system usability is the ability, in terms of human functionality, to be used easily and effectively by several users, with a certain level of training and support, to accomplish a specific range of tasks, within a specific series of scenarios. It can be seen that usability is therefore related to ease and efficiency of use. However, the most frequently cited definition is probably that of both standards ISO 9241-210 and ISO 9241-220 (ISO CD 9241-220, 2015) These standards specify that usability is the degree to which a service, product or system can be used by users to achieve specific goals with effectiveness, efficiency, and satisfaction in a specific use context. We see that this definition strongly mentions user satisfaction, but even with these definitions, usability is not a sufficiently encompassing concept to describe the experience with the product. Limiting user frustration is essential, but some products can also bring pleasure to users and make them feel different emotions. The concept of the user experience (UX) is dedicated to encompassing these aspects.

From the mid-1990s, the term UX appeared at Apple through Donald Norman (Norman, Miller and Henderson, 1995). In fact, the former explains that he proposed this term in order to encompass all the elements related to the experience with a product, including, among others, factors related to emotional and hedonic aspects. In the literature, one of the most frequently cited definitions is that of the two researchers Hassenzahl and Tractinsky (Hassenzahl and Tractinsky, 2011). These authors specify that UX is a consequence of characteristics of the designed system (e.g. usability, functionality, complexity, purpose, etc.), user's internal state (predispositions, motivation, needs, expectations, mood, etc.) and the context (or the environment) inside of which the interaction occurs (e.g. organizational/social setting, meaningfulness of the activity, voluntariness of use, etc.). They also point out that UX is strongly based on the idea of designing systems to please users rather than just to limit their frustrations.

In the field of e-learning, the literature indicates that the LX is designed to a large extent, such as the e-learner behaviour and satisfaction, or self-regulation and effort in learning, or even e-learner's knowledge (Ngamkamollert and Ruangkanjanases, 2015). In our previous research, we focused on the factors that evaluate LX in e-learning systems (Safsouf, Mansouri and Poirier, 2018). we proposed models to better understand the factors involved in measuring learner satisfaction, their intention to continue using the system, their self-regulation, their attitudes towards the same system and their success in online training.

\section{EVALUATION OF THE LEARNER'S EXPERIENCE}

To improve the user experience towards a system or simply to ensure that the final system has a good UX value, it is necessary to know how to evaluate it in a valid and reliable way. Self-assessment questionnaires are among the most commonly used instruments for evaluating UX. With UX scales, you will quickly know if your system is perceived by users as attractive, efficient, clear, reliable, challenging, or innovative.

UX evaluation scales are measurement tools used to collect user feedback and experience. They are in the form of questionnaires where users answer themselves, in order to evaluate users' perceptions of the system. They are currently used in all fields (Lallemand et al., 2015): video games, professional applications, health-related systems, virtual worlds and e-learning platforms. The AttrakDiff questionnaire is one of the most widely used user experience assessment tools at the academic level. This tool is based on the theoretical model proposed by Hassenzahl to explain the perceived quality of an interactive system. It includes 28 items divided into 4 subscales (pragmatic quality, hedonic quality-stimulation, hedonic quality-identity, overall attractiveness). The 28 items of the questionnaire are presented as semantic differentiators (pairs of antonymous words) in a 7 point scale (from -3 to +3 ). These scales are not graduated verbatim. The order in which the items are handed over is standardized.

In the field of e-learning, little research has been done on the experience of learners. Our contribution in this article is to propose a measurement instrument based on a theoretical model proposed in our previous studies in e-learning systems. We called it FASER LX for "Formation, Apprenant, Système, Enseignant, 
Relation Learner eXperience" (Course, Learner, System, Teacher, Relationship). Like the AttrakDiff, the FASER LX scale is self-administered, which means that users complete the questionnaire themselves. It consists of a total of 30 items presented as pairs of contrasting words, divided into 5 sub-scales:

- Pragmatic quality (PQ): with 9 items which describe the usability of the system and how it enables users to achieve their objectives;

- Hedonic-stimulation quality (HQ-ST): with 5 items which indicate the extent to which the system can support the need for stimulation;

- Hedonic-satisfaction quality (HQ-SA): with 4 items which indicate the extent to which the system allows the user to be satisfied;

- Effort quality (EQ): with 6 items which indicate the extent to which the system allows users to measure the effort deployed when using the system;

- Social quality (SQ): with 6 items which measure the appearance and social interactions of a user with the actors of the system (instructor and learners).

In FASER LX scale, each subscale has items where each represents a factor from our research and theoretical models. These factors have been previously classified by dimensions which are: learner characteristics, instructor characteristics, system characteristics, course characteristics and social aspects. In the following table we present the FASER LX scale, with the assignment items by dimensions and subscales.

Table 1. The items of the FASER LX scale to evaluate the learner experience in an e-learning system

\begin{tabular}{|c|c|c|c|}
\hline Dimensions & Subscales & Success model factors & Antonym pairs \\
\hline \multirow{7}{*}{$\begin{array}{c}\text { Learner } \\
\text { characteristics }\end{array}$} & PQ1 & Computer self-efficiency & Autonomous - Non-autonomous \\
\hline & HQ-SA1 & Self-enjoyment & Unpleasant - Pleasant \\
\hline & HQ-ST1 & Perceived usefulness & Boring - Captivating \\
\hline & EQ1 & Self-effort & Undemanding - Demanding \\
\hline & PQ2 & Self-regulation & Free use - Compulsory use \\
\hline & HQ-SA2 & Self-security & Confident - Distrustful \\
\hline & EQ2 & Perceived anxiety & Calming - Stressing \\
\hline \multirow{4}{*}{$\begin{array}{l}\text { Instructor } \\
\text { characteristics }\end{array}$} & SQ1 & Communication ability & Easy communication - Difficult communication \\
\hline & HQ-ST2 & Responsiveness & High reactivity - Low reactivity \\
\hline & EQ3 & Informativeness & Not comprehensible - Comprehensible \\
\hline & SQ2 & Fairness & Unfair - Highly fair \\
\hline \multirow{8}{*}{$\begin{array}{c}\text { System } \\
\text { characteristics }\end{array}$} & PQ3 & Connection access quality & Slow - Fast \\
\hline & EQ4 & Device and context independence & Device dependent - Device independent \\
\hline & PQ4 & Efficiency & Tedious - Efficient \\
\hline & PQ5 & Security & Unreliable - Very reliable \\
\hline & PQ6 & Perceived Ease-of-use & Difficult learning - Easy learning \\
\hline & PQ7 & Availability & Not available - Very available \\
\hline & HQ-ST3 & Interactivity & Not interactive - Very interactive \\
\hline & PQ8 & Personalization & Customizable - Not customizable \\
\hline \multirow{6}{*}{$\begin{array}{c}\text { Course } \\
\text { characteristics }\end{array}$} & EQ5 & Course Quality & Confused - Clear \\
\hline & HQ-ST4 & Content diversity & Not diversified - Very divided \\
\hline & EQ6 & Course Flexibility & Rigid - Flexible \\
\hline & HQ-SA3 & Design and system quality & Pleasant - Unpleasant \\
\hline & HQ-ST5 & Up-to-dateness & Static - Dynamic \\
\hline & PQ9 & Diversity in assessments & Diversified assessment - Not diversified assessment \\
\hline \multirow[t]{5}{*}{ Social aspects } & SQ3 & Subjective norm & Recommendable - Not recommendable \\
\hline & HQ-SA4 & Self-image & Valuable - Non-valuable \\
\hline & SQ4 & Learner-learner interaction & Gets closer to learners - Separates me from learners \\
\hline & SQ5 & Learner-instructor interaction & Get closer to teachers - Separate me from teachers \\
\hline & SQ6 & Instructor-instructor interaction & Bring teachers together - Separate teachers \\
\hline
\end{tabular}

The questionnaire is available for free online (www.safsouf.net/faserlx). However, only the French version is currently available. As well as for the AttrakDiff, to calculate a global score in the FASER LX scale, we must first reverse some items, that means, replace the score obtained by its opposite. Because sometimes the word on the left is negative and sometimes it is positive. The next step is to calculate the averages for each of the five sub-scales as well as the five dimensions. Finally, present the results as two graphs in radar form (see next figure). 


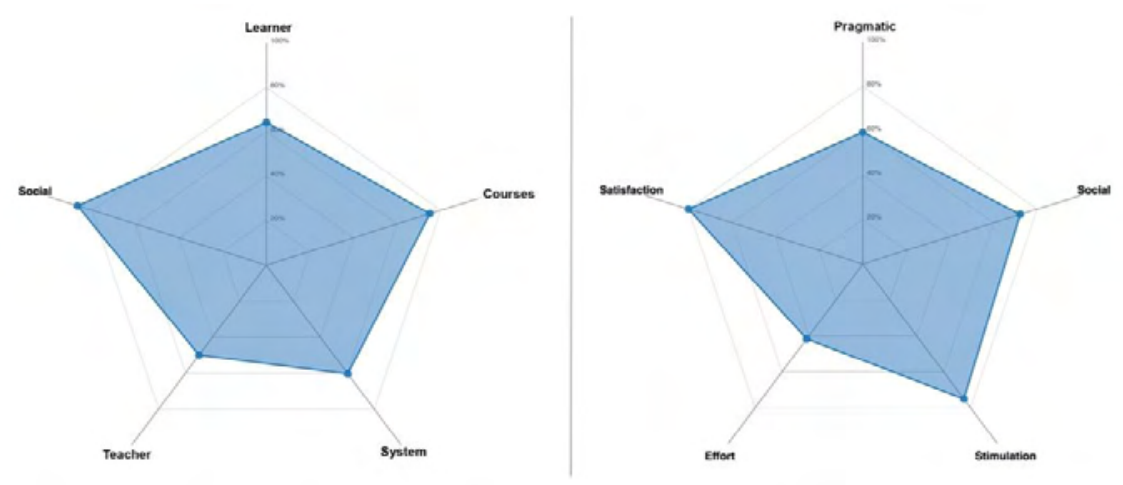

Figure 2. Presentation of the results of the FASER LX scale in graphical format

This graph shows the average percentage values for each pair of words. The items are grouped by dimensions in the left graph and by subscales in the right graph. This visualization makes it possible to quickly distinguish which aspects are perceived as critical and call for improvement actions or not.

\section{CONCLUSION}

In this work, we have attempted to present a new scale for measuring the learner experience in an online learning environment. Based on our previous research on success factors in understanding satisfaction, the intention to continue using the system, self-regulation and learner success in an online learning system and inspired by the AttrakDiff measurement scale, this scale is, according to our investigation, the first in the field of e-learning.

In our future work, we plan to validate this new scale of measurement in the context of higher education in Morocco.

\section{REFERENCES}

Hassenzahl, M. and Tractinsky, N. (2011) 'Behaviour \&amp; Information Technology User experience-a research agenda User experience-a research agenda'. doi: 10.1080/01449290500330331.

ISO CD 9241-220 (2015) 'Ergonomics of human-system interaction - Part 220: Processes for enabling, executing and assessing human-centred design within organizations'.

Lallemand, C. et al. (2015) 'Création et validation d'une version française du questionnaire AttrakDiff pour l'évaluation de l'expérience utilisateur des systèmes interactifs', Revue Europeenne de Psychologie Appliquee. Elsevier Masson SAS, 65(5), pp. 239-252. doi: 10.1016/j.erap.2015.08.002.

Ngamkamollert, T. and Ruangkanjanases, A. (2015) 'Factors Influencing Foreign Students' Satisfaction toward International Program in Thai Universities', International Journal of Information and Education Technology, 5(3), pp. 170-178. doi: 10.7763/IJIET.2015.V5.497.

Norman, D., Miller, J. and Henderson, A. (1995) CHI '95 MOSAIC OF CREATIVITY s Organization Overviews I What You See, Some of What's in the Future, And How We Go About Doing It: HI at Apple Computer.

Safsouf, Y., Mansouri, K. and Poirier, F. (2017) 'Towards a Multidimensional Model to Study a Critical Success Factors Affecting Continuity and Success in E-Learning Systems', Proceedings - International Conference on Developments in eSystems Engineering, DeSE, pp. 129-134. doi: 10.1109/DeSE.2017.26.

Safsouf, Y., Mansouri, K. and Poirier, F. (2018) 'A New Model of Learner Experience in Online Learning Environments', Information Systems and Technologies to Support Learning. Springer International Publishing, 111(Lx), pp. 29-38. doi: 10.1007/978-3-030-03577-8.

Shackel, B., Richardson, S. (1991) 'Usability—context, framework, design and evaluation', Human Factors for Informatics Usability. Cambridge University Press, Cambridge, pp. 21-38.

Sudhakar A., T. J. and W. J. (2015) 'Enhancing Student Experience and Performance through Peer-assisted Learning', Issues in Accounting Education Journal, 31(3), pp. 321-336. doi: 10.2308/iace-51249. 